\title{
Effect of nitrogen regime on microalgal lipid production during mixotrophic growth with glycerol
}

\author{
Kiran Paranjape ${ }^{1}$, Gustavo B. Leite ${ }^{1}$, and Patrick C. Hallenbeck ${ }^{1,2^{*}}$ \\ ${ }^{1}$ Département de Microbiologie, Infectiologie et Immunologie, Université de Montréal, CP6128 \\ Succursale Centre-ville, Montréal, Québec, Canada H3C 3J7 \\ ${ }^{2}$ Life Sciences Research Center, Department of Biology \\ United States Air Force Academy \\ 2355 Faculty Drive, USAF Academy, Colorado 80840
}

\begin{abstract}
*Corresponding author; Tel : (514)343-6278 Fax : (514)343-5701
E-mail address : patrick.hallenbeck@umontreal.ca
\end{abstract}

Mixotrophic growth of microalgae to boost lipid production is currently under active investigation. Such a process could be of practical importance if a cheap source of organic carbon, such as waste glycerol from biodiesel production, could be used. Several previous studies have already demonstrated that this carbon source can be used by different indigenous strains of microalgae. In this study it is shown that different nitrogen limitation strategies can be applied to further increase lipid production during growth with glycerol. In one strategy, cultures were grown in nitrogen replete medium and then resuspended in nitrogen free medium. In a second strategy, cultures were grown with different initial concentrations of nitrate. Lipid production by the two microalgal strains used, Chlorella sorokiniana (PCH02) and Chlorella vulgaris (PCH05), was shown to be boosted by strategies of nitrogen limitation, but they responded differently to how nitrogen limitation was imposed.

Keywords: microalgae; mixotrophic growth; lipid production; biofuels, nitrogen limitation 


\section{Introduction}

Biofuel production using microalgae has many promising characteristics, but at the same time there are many obstacles that must be overcome before this could be a practical process (Leite et al., 2013; Abedelaziz et al., 2013a, 2013b). One of the challenges to the practical development of algal biofuels is that growth conditions are suboptimal in many parts of the globe. For example, for countries like Canada in Northern latitudes ( $>45 \mathrm{~N}$ ), temperatures and solar flux would likely limit productivities over much of the year. Various strategies can be suggested for overcoming these challenges. To compensate for low temperatures, growth facilities could be located next to fossil fuel burning power plants to access the waste heat and carbon dioxide for enhancing algal growth. In addition, it is likely that indigenous algal strains, already adapted to the local climate, could show good growth, and perhaps lipid production, at lower temperatures than well studied strains. In fact, several recent studies have reported this to be the case, with a large survey of local algae showing that a wide range of growth rates at various temperatures can be obtained relatively easily (Abdelaziz, et al., 2014; Hallenbeck et al., 2014; Wang et al., 2016).

Different strategies are required to overcome the challenges posed by lower solar fluxes. One idea that is being explored is the use of heterotrophic growth of microalgae, or mixotrophic growth, where carbon dioxide fixation driven by photosynthesis is supplemented with organic carbon uptake and use. Organic carbon use by microalgae is species dependent, but as a whole, microalgae are capable of using a wide range of organic carbon compounds (Wang et al., 2014; Zhang et al., 2014). Of course, this kind of supplementation would be too costly, given the relatively low value of fuel, unless some form of waste stream were to be used. Fortunately, a number of possible waste streams have been proposed (Dubey et al., 2015; Lowrey et al., 2015; Mitra et al., 2012;). Heterotrophic cultivation is capable of producing relatively high biomass 
concentrations, and can be used as a second stage after a first stage of photoautotrophic growth (Rismani-Yazdi et al., 2015). However, high density bacterial or yeast fermentations are probably a better choice for using these substrates to make biofuels than a single stage heterotrophic process with algae (Qiao et al., 2015). In addition, two-stage algal systems require an intermediate stage of harvesting that add both complexity and cost to the process.

Hence, it would appear that the best use of low or no cost organic carbon substrates would be their use in supporting mixotrophic growth of microalgae. In at least some cases there appears to be a synergistic effect, with higher growth, biomass yields, and lipid productivities being obtained with mixotrophic growth than with photoautotrophic growth (Heredia-Arroyo et al., 2011; Lin et al., 2015; Woodworth et al., 2015). In fact, mixotrophic growth on glycerol can even exceed that seen with heterotrophic growth (Leite et al., 2015; Yeh and Chang, 2012). Crude glycerol is a byproduct of biodiesel production, and, given the present scale of biodiesel manufacture, so much glycerol is generated that it has become a waste disposal problem. Thus, glycerol could be an ideal substrate for mixotrophic growth.

Indeed, a number of recent studies have examined microalgal mixotrophic growth using glycerol as substrate (Leite, et al., 2015; Lin and Wu, 2015; Paranjape, et al., 2016; Skorupskaite et al., 2015; Wang et al., 2016). Various reports with different strains and species of Chlorella have found that the addition of glycerol can enhance final biomass or lipid yields or both. One study found up to a three-fold increase in dry weight with Chorella protothecoides growing with glycerol and limiting nitrogen (Skorupskaite et al., 2015). In another report, the addition of glycerol to an indigenous strain identified as Chorella gave a significant increase in both dry cell weight and lipid content (Lin and Wu, 2015). Similarly, it has been reported that mixotrophic growth of Chorella pyrenoidosa on glycerol gave a seven-fold increase in lipid content over that 
seen with photoautotrophic growth (Rai, et al., 2013). On the other hand, two studies with Chlorella vulgaris found that although mixotrophic growth with glycerol did not increase the cellular lipid content, lipid productivity was almost eight-fold higher than photoautotrophic growth due to a large increase in biomass productivity (Kong, et al., 2013; Liang et al., 2009). In our laboratory we have been investigating the mixotrophic growth of indigenous algae on glycerol and xylose, available in large quantities as a waste stream of the pulp and paper industry (Leite et al., 2015; Leite et al., 2016; Paranjape et al., 2016; Wang et al., 2016). We have found that many different strains are capable of using glycerol and that, in general, glycerol, when assimilated by a particular strain, boosts both cell biomass and lipid content.

A variety of previous studies have demonstrated that the lipid content of photoautotrophically grown microalgae can be increased through some form of stress (Leite et al., 2013; Breuer et al., 2013). In most cases, nitrogen limitation has been chosen as the stress used to induce lipid accumulation (Breuer et al., 2012; Negi et al., 2016, Griffiths et al., 2014). Fixed nitrogen is essential for the biosynthesis of a variety of required cellular components and hence cell growth is necessarily arrested in its absence. However, in the presence of light, photosynthetic metabolism continues to function and newly fixed carbon is accumulated as storage material since it cannot be used for cell growth in the absence of nitrogen. For example, resuspension of photoautotrophically grown cells of Chlorella sorokiniana in N-medium has been demonstrated to increase oil accumulation 20-fold (Negi et al, 2016). Several studies with Chlorella vulgaris have shown that the degree and timing of nitrogen limitation influence greatly how it affects lipid production (Griffiths et al., 2014; Stephenson et al., 2010). Since the addition of glycerol by itself seems to increase the lipid production of some strains, we were interested to see if some type of nitrogen limitation strategy with mixotrophic cultures could increase lipid production 
even further. Thus, in the present study, we examined the effects of two different nitrogen limitation strategies on the lipid production of several strains that were chosen based on their lipid production patterns as previously determined (Paranjape et al., 2016).

\section{Materials and Methods}

\subsection{Algal Cultivation}

The algal strains used in this study were from the collection of the Laboratory of Advanced Biofuels, Département de microbiologie, infectiologie et immunology of the Université de Montréal. These are strains isolated in the region of Québec, Canada, and were previously described (Abdelaziz et al., 2014, Hallenbeck et al., 2015)). Three strains were chosen for the present work; PCH02 (closely related to Chlorella sorokiniana), PCH05 (closely related to Chlorella vulgaris), and PCH 28 (closely related to Hindakia fallax). Bold's Basal medium (BBM) (Andersen, 2005) was used for photoautotrophic cultivation, and with $25 \mathrm{mM}$ glycerol, for mixotrophic cultivation. Strains were grown in a shaker in $125 \mathrm{ml}$ Erlenmeyer flasks containing $50 \mathrm{~mL}$ of $\mathrm{BBM}$ with and without $25 \mathrm{mM}$ glycerol. The strains were grown in constant light at $40 \mathrm{~W} / \mathrm{m}^{2}$ intensity at room temperature and shaken at 160rpm. Each day, $200 \mu$ l of culture were taken and placed in 96-well microplate for measurement of OD at 630nm using an EL-800 universal microplate reader from Bio-Tek instruments, Inc.. Maximal absorption for chlorophyll is at $675 \mathrm{~nm}$, and the region between 600 and $630 \mathrm{~nm}$ is a minimum in a spectral scan of a microalgal culture. In fact, this is the wavelength recommended by BioTek, a highly regarded manufacturer of microtiter plate readers (http://www.biotek.com/resources/articles/monitoringof-algal-growth-using-intrinsic-properties.html). For all experiments pre-inocula were grown under photoautotrophic conditions. Two different strategies to impose nitrogen limitation were used. In one strategy, cultures were first grown under photoautotrophic conditions in complete 
BBM for seven days, harvested by centrifugation, and then resuspended in medium lacking nitrogen with $25 \mathrm{mM}$ glycerol (mixotrophic conditions) and without glycerol (photoautotrophic conditions) and incubated for three weeks. $25 \mathrm{mM}$ glycerol was chosen since this is the concentration used in our previous study (Paranjape et al., 2016) and is similar to the concentration of glycerol used in several previous studies with this source of organic carbon (Lin and $\mathrm{Wu}, 2015$; Skorupskaite et al, 2015). In a second strategy, nitrogen limitation was imposed by using BBM medium with varying amounts of nitrate. As indicated, no, or varying amounts of sodium nitrate were used, to give a final nitrate concentration of $0 \mathrm{mg} \mathrm{L}^{-1}, 20 \mathrm{mg} \mathrm{L}^{-1}, 40 \mathrm{mg} \mathrm{L}^{-1}$, $60 \mathrm{mg} \mathrm{L}^{-1}$, 80 $\mathrm{mg} \mathrm{L}^{-1}, 100 \mathrm{mg} \mathrm{L}^{-1}$ and $250 \mathrm{mg} \mathrm{L}^{-1}$ (nitrogen-replete conditions).

\subsection{Biomass and Lipid Quantification}

Biomass (dry weight) was calculated using the relationship $\mathrm{OD}_{630}$ gm (dry wt $)^{-1}=1.055 \pm$ 0.12 previously determined (Abdelaziz et al., 2014). Nile red, a fluorescent dye capable of staining neutral lipids, was used to quantify intracellular lipids (Bertozzini et al, 2011) using a slight modification of previously used methods (Abdelaziz et al., 2014; Leite et al., 2015; Paranjape, et al., 2016). Algal samples were diluted with BBM to obtain 0.06 OD for each sample and $143 \mu 1$ was transferred into black flat-bottom 96 well plates. To this, $50 \mu 1$ of DMSO was added followed by the addition of $6.66 \mu \mathrm{l}$ of Nile red solution $\left(15 \mu \mathrm{g} \mathrm{m}{ }^{-1}\right.$ Nile red in acetone). The plate was incubated on a microplate shaker at room temperature in dim light for 10 minutes. The plates were read with a spectrofluorometer with excitation at $525 \mathrm{~nm}$ and emission at $580 \mathrm{~nm}$. A standard curve made with triolein was created each time samples were to be measured.

\subsection{Analytical and statistical procedures}

Glycerol was quantified using the colorimetric method described by Bondioli and Della Bella (2005) as previously modified for use with 96 well plates (Leite et al., 2015). All 
determinations were carried out with biological triplicates. Thus the results given in the various tables and figures are averages of three values. In most cases, standard deviations are indicated. In general these were within the range of 5 to $10 \%$.

\subsection{Growth Rate, Lipid Percentage and Lipid Productivity}

Growth rates were calculated according to the standard formula:

$$
\left.\mu=\frac{\ln (\mathrm{Xt} 1)-\ln (\mathrm{Xt} 2)}{\mathrm{t} 1-\mathrm{t} 2} \quad \text { (Eq. } 1\right)
$$

Where $\mu$ is the growth rate in days ${ }^{-1}, X_{t 1}$ and $X_{t 2}$ are the optical densities at time $t 1$ and $t 2$. For maximum growth rates, $\mathrm{X}_{\mathrm{t} 1}$ and $\mathrm{X}_{\mathrm{t} 2}$ were taken as the point at which cultures enter and complete exponential growth.

Lipid content was calculated as the ratio of the total amount of lipids produced to the total amount of biomass produced by a culture:

$X(\%)=\frac{X(T)}{Y} \times 100 \quad$ (Eq. 2)

Where $\mathrm{X}(\%)$ is the percent lipid, $\mathrm{X}(\mathrm{T})$ is total lipid, $\mathrm{Y}$ is total biomass.

Lipid productivity is defined as the lipid yield per volume of growth medium per unit time and is expressed as $\mathrm{mg} \mathrm{L}^{-1} \mathrm{~d}^{-1}$. Thus, to calculate lipid productivity the following formula was used:

$Z=\frac{X(T)}{\mathrm{V} * \mathrm{~N}}(\mathrm{Eq} .3)$

Where $\mathrm{Z}$ is lipid productivity, $\mathrm{X}(\mathrm{T})$ is total lipid $(\mathrm{mg}), \mathrm{V}$ is the volume of the culture (l) and $\mathrm{N}$ is the number of days

\section{Results and discussion}

\subsection{Nitrogen depletion and accumulation of neutral lipids}

The pattern of lipid accumulation in the presence and absence of glycerol when the cells were subjected to nitrogen deprivation was determined. Three different strains from the previous screen (Paranjape et al., 2016) were selected for further analysis based on their lipid production 
patterns. Strain PCH02 had previously shown both a good growth rate and significant biomass production when grown with glycerol. It also had, out of all of the strains tested, the largest lipid increase under mixotrophic conditions, and had shown an interesting pattern of responses when examined by flow cytometry. Strain PCH05, in addition to also showing an increase in lipid production in presence of glycerol, had shown the highest lipid production for both growth conditions, and had given a different pattern of responses in comparison to $\mathrm{PCH} 02$ when examined by flow cytometry. In addition, phylogenetic analysis had shown that this strain is closely associated with Chlorella vulgaris and phylogenetically distinct from strain $\mathrm{PCH} 02$, which is more closely associated to Chlorella sorokiniana. Finally, strain $\mathrm{PCH} 28$, which belongs to the Hindakia genus, being most closely related to Hindakia fallax, was selected as a strain which had shown a different pattern when screened in that, unlike strains $\mathrm{PCH} 02$ and PCH05, it had only low lipid production under the growth conditions tested above.

One strategy for inducing nitrogen limitation is to first grow cultures under nitrogen replete conditions and then resuspend them in medium lacking nitrogen. Thus, active cultures with appreciable amounts of biomass were obtained by growing the selected strains under nitrogen-replete photoautotrophic conditions for seven days at which point they had attained ODs ranging from $0.63 \pm 0.041$ to $0.88 \pm 0.019\left(0.66 \pm 0.045\right.$ to $\left.0.93 \pm 0.02 \mathrm{~g} \mathrm{~L}^{-1}\right)$. The cultures were then resuspended in medium lacking nitrogen with $25 \mathrm{mM}$ glycerol (mixotrophic conditions) and without glycerol (photoautotrophic conditions) and incubated for three weeks. When changes in OD (optical density) were followed, two different phases can be observed (Figure 1A). There was an initial phase, lasting from inoculation to the first day and a half where additional growth occurred. For example, strain PCH02 had an OD of 0.74 at inoculation, but after 36 hours the OD had increased to 1.34. After this initial period of growth, the strains entered a phase where the OD was stationary and no additional growth occurred. It is reasonable 
to assume that, since the algal cultures had been grown under nitrogen-replete conditions, sufficient nitrogen stores were available after resuspension in nitrogen-free medium to permit one or two cell doublings before growth arrest due to nitrogen depletion. A similar observation was made in a previous study when $C$. sorokinia was resuspended in a N- medium (Negi et al., 2016).

When total lipid production over the period of incubation under nitrogen-free conditions is examined a number of interesting observations can be made. First, strain $\mathrm{PCH} 28$ showed very little increase in lipid production throughout the nitrogen starvation period under both conditions, essentially staying constant (Figure 1B). In fact, lipid production with this strain under the nitrogen deprivation conditions used here was only slightly higher than that observed under the conditions used for the initial screening. Thus, it would appear that strain $\mathrm{PCH} 28$ did not respond to the nitrogen stress applied here, and, in accordance with this, this strain remained green throughout the incubation period, therefore showing no signs of the chlorophyll degradation that is usually induced by nitrogen starvation.

On the other hand, by the end of the incubation period, strain PCHO2 (C. sorokina) had shown a marked response to incubation in nitrogen-free medium with increased lipid production over the period of nitrogen starvation for both mixotrophic (13 fold) and photoautotrophic conditions (12 fold) (Figure 1B). Lipid production under both conditions increased very little for the first 10 days, dramatically increasing afterwards reaching levels as high as $0.3 \pm 0.025$ to $0.4 \pm 0.02 \mathrm{~g} \mathrm{~L}^{-1}$. Again, this agrees with a previous report of greatly increased oil accumulation by a strain of $C$. sorokinia when resuspended in N- medium (Negi et al., 2016).

Strain PCH05 (C. vulgaris) also showed a lipid production response to nitrogen limitation. When examined under the same conditions, strain PCH05 gave a large difference in total lipid production, accumulating lipids to much higher levels in the presence of glycerol than in its absence, $0.51 \pm 0.026 \mathrm{~g} \mathrm{~L}^{-1}$ versus $0.102 \pm 0.006 \mathrm{~g} \mathrm{~L}^{-1}$ (a five-fold difference (Fig 1B). This is 
somewhat surprising since this was not the case in the previous study where the cultures were not subjected to a sudden nitrogen limitation (Paranjape et al. 2016). However, a significant portion of this difference was due to higher cell densities when the cells were incubated as indicated, with final biomass concentrations of $2.47 \pm 0.12 \mathrm{~g} \mathrm{~L}^{-1}\left(\mathrm{M}\right.$, mixotrophic) and $2.37 \pm 0.085 \mathrm{~g} \mathrm{~L}^{-1}(\mathrm{~A}$, autotrophic), $\mathrm{PCH} 02 ; 2.55 \pm 0.18 \mathrm{~g} \mathrm{~L}^{-1}(\mathrm{M})$ and $2.42 \pm 0.21 \mathrm{~g} \mathrm{~L}^{-1}(\mathrm{~A}), \mathrm{PCH} 05 ; 1.35 \pm 0.15 \mathrm{~g} \mathrm{~L}^{-1}(\mathrm{M})$ and $2.1 \pm 0.024 \mathrm{~g} \mathrm{~L}^{-1}$ (A) $\mathrm{PCH} 28$. The relatively small increase in lipid content under these conditions of photoautotrophic cultures of strain PCH05 (C. vulgaris) agrees with earlier reports carried out with strains of $C$. vulgaris (Mujtiba et al., 2012; Stephenson et al., 2010; Griffiths et al., 2014).

Thus, when these three strains were resuspended in nitrogen-free medium, subsequent incubation under nitrogen starvation had different effects on lipid production which seemed to give species-specific responses and to be different for the two different conditions. The direct inoculation of algal samples into nitrogen depleted medium might have contributed to relatively low lipid yields for some of the strains since some algal species produce greater lipid quantities when nitrogen concentrations are depleted naturally. Therefore, a series of experiments were undertaken to ascertain the effect of growing selected strains on different initial limiting concentrations of nitrate.

\subsection{Variation in biomass production and growth rate for strains PCHO2 and PCHO5 grown with}

\section{different nitrate concentrations}

A previous study with $C$. vulgaris showed that this alga was more effective at producing high quantities of lipids when cells depleted their nitrogen source naturally instead of being transferred directly into a nitrogen depleted medium (Stephenson et al, 2010). A similar experiment was conducted with $\mathrm{PCH} 02$ and $\mathrm{PCH} 05$, where these strains were grown with different initial nitrate concentrations under either mixotrophic conditions with $25 \mathrm{mM}$ glycerol or photoautotrophic 
conditions in its absence. Cultures with $250 \mathrm{mg} \mathrm{L}^{-1}$ nitrate correspond to the "nitrogen-replete" conditions of normal BBM, a very commonly used medium for microalgal growth.

The two strains presented different responses in terms of maximal growth rates and biomass productivities under mixotrophic and photoautotrophic conditions (Table 1 and Figure 2). For strain PCH05, the maximal growth rate was essentially indifferent to the presence (M, mixotrophy) or absence (P, photoautotrophy) of glycerol and was the same between $20 \mathrm{mg} \mathrm{L}^{-1}$ and $250 \mathrm{mg} \mathrm{L}^{-1}$ nitrate. Thus, between a nitrate concentration of $20 \mathrm{mg} \mathrm{L}^{-1}$ and $250 \mathrm{mg} \mathrm{L}^{-1}$, the average growth rate was around $0.632 \mathrm{~d}^{-1}$ and $0.637 \mathrm{~d}^{-1}$ for mixotrophic and photoautotrophic conditions, with less than a ten percent variation for the different nitrate concentrations. On the other hand, strain PCH02 had higher growth rates under mixotrophic than under photoautotrophic conditions, with increases up to $65 \%$ (at $250 \mathrm{mg} \mathrm{L}^{-1}$ nitrate concentration). Furthermore, maximum growth rates increased as nitrate concentration increased under mixotrophic conditions, but remained constant $\left(0.785 \mathrm{~d}^{-1}\right.$ on average $)$ between a nitrate concentration of 20 and $250 \mathrm{mg} \mathrm{L}^{-1}$ for photoautotrophic conditions, with less than ten percent difference (Table 1).

For both strains, total biomass increased under both growth conditions as nitrate concentrations increased (Table 1 and Figure 2). This suggests that growth yields are directly dependent upon the concentration of nitrate and thus, nitrogen-replete conditions had the highest biomass production. In fact, a plot of final biomass versus initial nitrate concentration directly shows that biomass increases linearly with initial nitrate concentration, as shown in Figure 3 for strain PCH05, regardless of whether mixotrophic or photoautotrophic growth is examined This is a relatively well known phenomenon and has been observed in other studies (e.g. Figure 5, Stephenson et al., 2010).

Interestingly enough, although at some nitrate concentrations the differences were small, strain PCH05 consistently produced higher biomass under mixotrophic conditions under all 
nitrate concentrations, whereas strain $\mathrm{PCHO} 2$ only had higher biomass production under mixotrophic conditions starting at $60 \mathrm{mg} \mathrm{L}^{-1}$ nitrate (Figure 2). Since glycerol was consumed by both strains, these findings suggest that both these two strains assimilate glycerol in different ways. (In the experiment shown in Figure 1, strain PCH02 consumed 51\% and strain PCH02 consumed $47 \%$ of the initial glycerol.)

Strain PCH02 had higher maximum growth rates and higher biomass production under mixotrophic conditions implying that this strain uses glycerol for growth and quite probably for lipid production. Strain PCH05 had essentially the same growth rate under both conditions, but increased total biomass production under mixotrophic conditions, suggesting that this strain might use glycerol directly for production of storage compounds, such as TAGs. Thus, these findings suggest that strains $\mathrm{PCH} 05$ and $\mathrm{PCH} 02$ were directing glycerol assimilation to lipid production and therefore it was of interest to further investigate lipid production by these two strains.

\subsection{Lipid production of strains $\mathrm{PCHO2}$ and PCHO5 with different initial nitrogen concentrations} under photoautotrophic and mixotrophic growth modes

The two strains were grown under both mixotrophic and photoautotrophic conditions with varying initial concentrations of nitrate and neutral lipid measurements were conducted using a Nile red assay. For both strains, added glycerol (mixotrophic conditions) appeared to positively affect lipid productivities (Figure 4), percentage lipid content (Figure 5), and total volumetric lipid production (Figure 6). That is, these values were equal to or greater than those seen for the same strain grown under photoautotrophic conditions, with the greatest effects seen at $40 \mathrm{mg} \mathrm{L}^{-1}$ nitrate or greater. The response of both strains to different nitrogen concentrations were qualitatively similar under photoautotrophic conditions, and corresponded to the consensus notion that fixed nitrogen levels greatly affect lipid content and lipid productivities. 
However, when examined in a number of different ways, either in terms of total lipid production (Figure 4), percent lipid content (Figure 5), or total volumetric lipid production (Figure 6), the two microalgal strains were quite different in terms of how lipid production and content were affected by the presence of glycerol. Specific lipid production (lipid production per volume of culture per density of culture) also increased with time for both strains (results not shown). This indicates that algal strains accumulate lipid throughout growth. When examined at the end of the incubation period, it can be seen that mixotrophic growth induced higher levels of lipid productivity than photoautotrophic conditions for the two algal strains (Figure 4).

Furthermore, lipid productivity was different for mixotrophic and photoautotrophic growth conditions for strain $\mathrm{PCH02}$ (C. sorokinia), whereas strain $\mathrm{PCH} 05$ (C. vulgaris) had similar productivity patterns under both conditions. Under mixotrophic conditions, the productivity levels of strain PCH02 increased as nitrate levels increased, with the highest productivity (40.3 $\pm 0.40 \mathrm{mg} \mathrm{L}^{-1} \mathrm{~d}^{-1}$ ) at $250 \mathrm{mg} \mathrm{L}^{-1}$ (nitrogen replete conditions) (Figure 4). However, under photoautotrophic conditions, lipid productivity decreased as the nitrate concentration increased, with the highest lipid productivity $\left(11.5 \mathrm{mg} \pm 0.44 \mathrm{~L}^{-1} \mathrm{~d}^{-1}\right)$ at $20 \mathrm{mg} \mathrm{L}^{-1}$. As far as we know, this is the first study of this type with a strain of $C$. sorokinia. Strain PCH05 (C. vulgaris) presented a much different picture. In contrast to strain $\mathrm{PCH} 02$, the highest productivity of strain $\mathrm{PCH} 05$ was found at $80 \mathrm{mg} \mathrm{L}^{-1}$ of nitrate for both mixotrophic and photoautotrophic conditions (Figure 4). Mixotrophic growth induced higher lipid productivity, $21.7 \pm 3.31 \mathrm{mg} \mathrm{L}^{-1} \mathrm{~d}^{-1}$, than photoautotrophic conditions, $15.9 \pm 5.94 \mathrm{mg} \mathrm{L}^{-1} \mathrm{~d}^{-1}$. Higher and lower concentrations of nitrate supported progressively smaller productivities. This is similar to a previous report with photoautotrophically grown $C$. vulgaris where maximum productivity was found at $170 \mathrm{mg} \mathrm{L}^{-1}$ nitrate (Griffiths et al., 2014). 
Moreover, the results shown in Figure 5 suggest that nitrogen starvation does not significantly affect lipid accumulation under mixotrophic conditions, but, does induce lipid accumulation under photoautotrophic conditions. For example, examining the lipid content of strain PCH02 it can be seen that the percent lipid under mixotrophic conditions is very similar (around 30\%), less than ten percent difference, for each of the different nitrate concentration (Figure 5). In contrast, under photoautotrophic conditions, the lipid content was highest, at $33 \%$, for growth at $20 \mathrm{mg} \mathrm{L}^{-1}$ nitrate, with a gradual decrease in lipid content as the nitrate concentration increased.

As can be seen from the results (Figure 6), total lipid production for both strains was consistently higher under mixotrophic growth conditions than under photoautotrophic conditions. Under mixotrophic conditions, the highest neutral lipid levels $\left(0.32 \mathrm{~g} \pm 0.0032 \mathrm{~g} \mathrm{~L}^{-1}\right.$ and $\left.0.37 \pm 0.056 \mathrm{~g} \mathrm{~L}^{-1}\right)$ were produced in stationary phase for strain PCH02 (C. sorokiniana) and strain PCH05 (C. vulgaris) respectively (Figure 6). In contrast, under photoautotrophic conditions, maximal lipid production at stationary phase only reached $0.034 \pm 0.0027 \mathrm{~g} \mathrm{~L}^{-1}$ for strain $\mathrm{PCH} 02$ whereas it was $0.323 \pm 0.048 \mathrm{~g} \mathrm{~L}^{-1}$ for strain PCH05. Thus, strain PCH02 showed, at $250 \mathrm{mg} \mathrm{L}^{-1}$ $\mathrm{NaNO}_{3}$, a remarkable ten-fold difference in lipid production depending upon whether or not glycerol was present. This was due to a very significant difference in how lipid production of this strain responded to nitrogen limitation in the presence of glycerol (mixotrophic conditions) or its absence (photoautotrophic conditions). Total lipid production under mixotrophic conditions increased monotonically with initial nitrate concentration whereas under photoautotrophic conditions, total lipid production was maximal at $20 \mathrm{mg} \mathrm{L}^{-1} \mathrm{NaNO}_{3}$ with a monotonic decline as nitrate concentrations were increased (Figure 6).

On the other hand, the total lipid production patterns of strain PCH05 were very different with essentially the same response under both mixotrophic and photoautotrophic conditions, an increase in total lipid production with increasing nitrate concentrations (Figure 6). Under 
mixotrophic conditions, the highest total lipid production was reached at $80 \mathrm{mg} \mathrm{L}^{-1}$ nitrate, with $0.369 \mathrm{~g} \mathrm{~L}^{-1}$ of lipid produced. At higher or lower concentrations of nitrate, lipid production decreased. Essentially the same pattern is seen for photoautotrophic conditions, however, at $250 \mathrm{mg} \mathrm{L}^{-1}$, total lipid production is higher than at $80 \mathrm{mg} \mathrm{L}^{-1}$. The increase in lipid production at nitrogen-replete conditions is probably due to the higher biomass production, since total lipid production is a function of both lipid content and biomass. As discussed earlier, biomass production increased with higher nitrate concentrations. Thus, for nitrogen-replete conditions (250 $\mathrm{mg} \mathrm{L}^{-1}$ nitrate), the lipid content was lower (Figure 5) but the total biomass produced was higher, causing similar production levels as that of $80 \mathrm{mg} \mathrm{L}^{-1}$. As a general rule, growth at lower nitrogen concentrations, between $0 \mathrm{mg} \mathrm{L}^{-1}$ and $80 \mathrm{mg} \mathrm{L}^{-1}$, increased the lipid content, but lowered total biomass production (Figure 2), resulting in lower total lipid production levels at low nitrate concentrations. A trade-off seems to be found at $80 \mathrm{mg} \mathrm{L}^{-1}$ nitrate, where the lipid content and biomass are high enough to give the same total lipid as nitrogen-replete conditions.

The differences noted between the two species of Chlorella are intriguing. While the differences shown above were obtained at stationary phase, it is very interesting to note that the same qualitative variation is seen with cultures at intermediate points of cultivation, for example, near the end of rapid growth (Figure S1). Therefore, the divergence in the two responses must reflect significant differences at the physiological and biochemical levels. However, while the mechanism(s) of response to nitrogen limitation by microalgae are of general interest, they are only poorly understood at present. In Chlamydomonas reinhardtii, three acyltransfereases and a potential transcriptional regulator have been shown to be upregulated in response to nitrogen limitation (Boyle et al., 2012). In contrast to this relatively specific response, RNA-Seq analysis has shown that the model diatom, Phaeodactylum tricornutum, responds to nitrogen limitation by upregulating the expression of 1213 genes, including those for carbon fixation, the TCA cycle, 
glycerolipid metabolism and nitrogen assimilation (Yang et al., 2013). Similarly, the microalga Neochloris oleoabundans appears to undergo extensive metabolic remodeling in response to nitrogen limitation with the overexpression of the fatty acid synthesis pathway, repression of the $\beta$-oxidation pathway, and up-regulation of the pentose phosphate pathway and the pyruvate dehydrogenase complex (Rismani-Tazdi et al., 2012). Further detailed work on the molecular level would be required to understand the metabolic differences in the two Chlorella strains noted here.

3.4. Comparison of lipid production by strains PCHO2 and PCHO5 under the two different nitrogen regimes

Here we have examined two different nitrogen regimes to assess their effects on neutral lipid accumulation in two strains; PCH02 (Chlorella sorokiniana) and PCH05 (Chlorella vulgaris) incubated under mixotrophic conditions (with glycerol) and under photoautotrophic conditions. Both treatments, strategy 1, growth under nitrogen replete conditions followed by resuspension in nitrogen free medium (Section 3.1, Figure 1), or, strategy 2, growth with different initial nitrate concentrations (Sections 3.2, 3.3, Figures 2-6), induced in some cases an increase in lipid content and, sometimes, an increase that was augmented by the presence of glycerol. Interestingly, the presence of glycerol had a greater impact on lipid accumulation by strain PCH05 when strategy 1 was used (Figure1, resuspension in $\mathrm{N}$-free BBM) whereas it was strain PCH02 that showed the greatest response to the presence of glycerol when strategy 2 (Figure 6, growth on limiting nitrogen) was employed. This highlights the fact that these two closely related, but distinct, species show marked differences in their response to nitrogen limitation when growing mixotrophically with glycerol. 
Given that both strategies appear to be capable of producing cultures with a high lipid content and an overall high total lipid yield, which is to be preferred? Resuspension in nitrogen free medium with glycerol, strategy 1, gave higher total lipids for both PCH02 and PCH05 (compare Figures 1 and 6). On the other hand, the amounts of total lipid are only somewhat higher than what was found with growth on limiting nitrate, an advantage that is far outweighed by other considerations. For one thing, strategy 1 employs a centrifugation step prior to resuspension, a process that would be too costly in terms of energy input for a practical process. Moreover, strategy 1 requires extended incubation times, with seven days in the initial growth phase and then fifteen to twenty in the second, lipid accumulation phase, giving a total time requirement from inoculation to harvest of twenty-two to twenty-seven days. In this regard, growth with limiting nitrate is capable of reaching nearly the same levels of total lipid, with only seven (PCH02) to fourteen days (PCH05) between inoculation and harvest (Figure S2). Thus, this second strategy would offer a substantial savings in both CAPEX (capital expenditures) and OPEX (operating expenses) over strategy 1.

\section{Conclusion}

Two different nitrogen limiting strategies to boost lipid production in the presence of glycerol were examined with two different species of Chlorella. Growth with limiting nitrate concentrations, a strategy that is more favorable in terms of both OPEX and CAPEX, was able to support high levels of total lipid production over relatively short periods of time (one to two weeks). Therefore, mixotrophic growth using glycerol, a waste product of the biodiesel industry, can be considered for industrial production of biodiesel. Lipid production by the two strains had different responses to nitrogen limitation, suggesting very interesting fundamental physiological differences. 


\section{Acknowledgments}

This research was supported by a grant from FQRNT (Le Fonds Québécois de la recherche sur la nature et les technologies), Programme de recherche en partenariat contribuant à la séquestration des gaz à effet de serre (2011-GZ-141307) to P.C.H. and by Biorémédiation d'effluents industriel provenant de l'industrie des biocarburants via la culture de biomasse algale mixotrophe (Numéro de référence : 24198) and CRSNG-STPGP 447266-13 Bio-oil Recovery \& CO2 Recycling by Waste Stream Enhanced Microalgal Growth \& Low Energy CO2-Related Extraction.

PCH holds a National Research Council Senior Research Associateship Award at the Life Sciences Research Center, Department of Biology, United States Air Force Academy. The views expressed in this article are those of the authors and do not reflect the official policy or position of the United States Air Force, the Department of Defense, or the US Government.

\section{References}

1) Abdelaziz, A. E. M, Leite, G. B., and Hallenbeck, P. C. 2013a. Addressing the challenges for sustainable production of algal biofuels: I. Algal strains and nutrient supply. Environ. Technol. 34: 1783-1805.

2) Abdelaziz, A. E. M, Leite, G. B., and Hallenbeck, P. C. 2013b. Addressing the challenges for sustainable production of algal biofuels: II. Harvesting and conversion to biofuels. Environ. Technol. 34: 1807-1836.

3) Abdelaziz, A.E.M., Leite, G.B., Belhaj, M.A., Hallenbeck, P.C. 2014. Screening microalgae native to Quebec for wastewater treatment and biodiesel production. Bioresour. Technol., 157, 140-148. 
4) Bertozzini, E., Galluzzi, L., Penna, A., and Magnani, M. 2011. Application of standard addition method for the absolute quantification of neutral lipids in microalgae using Nile Red. J. Microbiol. Methods. 87: 17-23.

5) Bondioli, P., and Della Bella, L. 2005. An alternative spectrophotometric method for the determination of free glycerol in biodiesel. Eur. J. Lipid Sci. 107: 153-157.

6) Boyle, N.R., Page, M.D., Liu, B., Blaby, I.K., Casero, D., Kropat, J., Cokus, S.J., HongHermesdorf, A., Shaw, J., Karpowicz, S. J., Gallaher, S.D., Johnson, S., Benning, C., Pellegrini, M., Grossman, A., and Merchant, S.S. 2012. Three Acyltransferases and Nitrogen-responsive Regulator are implicated in Nitrogen Starvation-induced Triacylglycerol Accumulation in Chlamydomonas. J. Biol. Chem. 287, 15811-15825.

7) Breuer, G., Lamers, P.P., Martens, D.E., Draaisma, R.B., Wijffels, R.H., 2012. The impact of nitrogen starvation on the dynamics of triacylglycerol accumulation in nine microalgae strains. Bioresour. Technol. 124, 217-226.

8) Breuer, G., Lamers, P.P., Martens, D.E., Draaisma, R.B., Wijffels, R.H. 2013. Effect of light intensity, $\mathrm{pH}$ and temperature on triacylglycerol (TAG) accumulation induced by nitrogen starvation in Scenedesmus obliquus. Bioresour. Technol. 143, 1-9.

9) Dubey, K., Kumar, S., Dixit, D., Kumar, P., Kumar, D., Jawed, A., Haque, S. 2015. Implication of Industrial Waste for Biomass and Lipid Production in Chlorella minutissima under Autotrophic, Heterotrophic, and Mixotrophic Grown Conditions. Appl. Biochem. Biotechnol. 176, 1581-1595.

10) Griffiths, M.J., van Hille, R.P., Harrison, S.T.L. 2014. The effect of nitrogen limitation on lipid productivity and cell composition in Chlorella vulgaris. Appl. Microbiol. Biotechnol. $98: 2345-2356$. 
11) Hallenbeck, P.C., Leite, G.B., Abdelaziz, A.E.M. 2014. Exploring the diversity of microalgal physiology for applications in wastewater treatment and biofuel production. Algal Research-Biomass Biofuels and Bioproducts. 6:111-118.

12) Heredia-Arroyo, T., Wei, W., Ruan, R., and Hu, B. 2011. Mixotrophic cultivation of Chlorella vulgaris and its potential application for the oil accumulation from non-sugar materials. Biomass and Bioenergy. 35: 2245-2253.

13) Kong, W. B., Yang, H., Cao, Y. T., Song, H., Hua, S. F., Xia, C. G. 2013. Effect of glycerol and glucose on the enhancement of biomass, lipid and soluble carbohydrate production by Chlorella vulgaris in mixotrophic culture. Food Technol. Biotechnol. 51: 62-69.

14) Leite, G.B., Abdelaziz, A.E.M., Hallenbeck, P.C. 2013. Algal biofuels: Challenges and opportunities. Bioresour. Technol. 145:134-141.

15) Leite, G.B., Paranjape, K., Hallenbeck, P.C. 2016. Breakfast of Champions: Fast Lipid Accumulation by Cultures of Chlorella and Scenedesmus induced by Xylose, Algal Res., in press

16) Leite, G.B., Paranjape, K., Abdelaziz, A.E.M., Hallenbeck, P.C. 2015. Utilization of biodiesel-derived glycerol or xylose for increased growth and lipid production by indigenous microalgae. Bioresour. Technol. 184, 123-130.

17) Liang, Y., Sarkany, N., and Cui, Yi. 2009. Biomass and Lipid productivities of Chlorella vulgaris under autotrophic, heterotrophic and mixotrophic growth conditions. Biotechnol. Lett. 31:1043-1049.

18) Lin, T.-S., Wu, J.-Y. 2015. Effect of carbon sources on growth and lipid accumulation of newly isolated microalgae cultured under mixotrophic condition. Bioresour. Technol. $184: 100-107$ 
19) Lowrey, J., Brooks, M., McGinn, P. 2015. Heterotrophic and mixotrophic cultivation of microalgae for biodiesel production in agricultural wastewaters and associated challenges-a critical review. J. Appl. Phycol. 27, 1485-1498.

20) Mitra, D., van Leeuwen, J., Lamsal, B. 2012. Heterotrophic/mixotrophic cultivation of oleaginous Chlorella vulgaris on industrial co-products. Algal Res. 1, 40-48.

21) Mujtaba, G., Choi, W., Lee, C-G., and Lee, K. 2012. Lipid production by Chlorella vulgaris after a shift from nutrient-rich to nitrogen starvation conditions. Bioresour. Technol. 123: 279-283.

22) Negi, S., Barry, A.N., Friedland, N., Sudasinghe, N., Subramanian, S., Pieris, S., Holguin, F.O., Dungan, B., Schaub, T., Sayre, R. 2015. Impact of nitrogen limitation on biomass, photosynthesis, and lipid accumulation in Chlorella sorokiniana. J. Appl. Phycol. 28:803812.

23) Paranjape, K., Leite, G.B., Hallenbeck, P.C. 2016. Strain variation in microalgal lipid production during mixotrophic growth with glycerol. Bioresour. Technol., 204, 80-88.

24) Qiao, K., Imam Abidi, S.H., Liu, H., Zhang, H., Chakraborty, S., Watson, N., Kumaran Ajikumar, P., Stephanopoulos, G. 2015. Engineering lipid overproduction in the oleaginous yeast Yarrowia lipolytica. Metabolic Engineering, 29, 56-65.

25) Rai, M. P, Nigam, S., and Sharma, R. 2013. Response of growth and fatty acid compositions of Chlorella pyrenoidosa under mixotrophic cultivation with acetate and glycerol for bioenergy application. Biomass Bioenergy. 58: 251-257.

26) Rismani-Yazdi, H., Haznedaroglu, B.Z., Hsin, C., and Peccia, J. 2012. Transcriptomic analysis of the oleaginous microalga Neochloris oleoabundans reveals metabolic insights into triacylglyceride accumulation Biotechnol. Biofuels 5:74 
27) Rismani-Yazdi, H., Hampel, K.H., Lane, C.D., Kessler, B.A., White, N.M., Moats, K.M., Allnutt, F.C.T. 2015. High-productivity lipid production using mixed trophic state cultivation of Auxenochlorella (Chlorella) protothecoides. Bioprocess and Biosystems Engineering, 38:639-650.

28) Skorupskaite, V., Makareviciene, V., Levisauskas, D. 2015. Optimization of mixotrophic cultivation of microalgae Chlorella sp for biofuel production using response surface methodology. Algal Res.-Biomass Biofuels Biopro. 7:45-50.

29) Stephenson, A.I., Dennis, J.S., Howe, C.J., Scott, S.A., Smith, A.G. 2010. Influence of nitrogen-limitation regime on the production by Chlorella vulgaris of lipids for biodiesel feedstocks. Biofuels. 1:47-58.

30) Wang, J., Yang, H., Wang, F. 2014. Mixotrophic Cultivation of Microalgae for Biodiesel Production: Status and Prospects. Appl. Biochem. Biotechnol. 172:3307-3329

31) Wang, Y.-Z., Hallenbeck, P.C., Leite, G.B., Paranjape, K., Huo, D.-Q. 2016. Growth and lipid accumulation of indigenous algal strains under photoautotrophic and mixotrophic modes at low temperature. Algal Research, 16:195-200.

32) Woodworth, B.D., Mead, R.L., Nichols, C.N., Kolling, D.R.J. 2015. Photosynthetic light reactions increase total lipid accumulation in carbon-supplemented batch cultures of Chlorella vulgaris. Bioresour. Technol. 179:159-164.

33) Yang, Z.-K., Niu, Y.-F., Ma, Y.-H., Xue, J., Zhang, M.-H., Yang, W.-D., Liu, J.-S., Lu, S.-H., Guan, Y., Li, H.Y. 2013. Molecular and cellular mechanisms of neutral lipid accumulation in diatom following nitrogen deprivation. Biotechnol. Biofuels 6:67

34) Yeh, K.-L., Chang, J.-S. 2012. Effects of cultivation conditions and media composition on cell growth and lipid productivity of indigenous microalga Chlorella vulgaris ESP-31. Bioresour. Technol. 105:120-127. 
35) Zhang, W., Zhang, P., Sun, H., Chen, M., Lu, S., Li, P. 2014. Effects of various organic carbon sources on the growth and biochemical composition of Chlorella pyrenoidosa. Bioresour. Technol. 173:52-58.

\title{
Figure Legends
}

\section{Figure 1: Optical density changes and specific lipid production of strains PCH02, PCH05 and PCH28 incubated in N-BBM}

\begin{abstract}
Algal cultures were resuspended in N-BBM with (mixotrophic) and without (photoautotrophic) $25 \mathrm{mM}$ glycerol and incubated with constant shaking $(150 \mathrm{rpm})$ at room temperature under constant illumination $\left(40 \mathrm{~W} / \mathrm{m}^{2}\right)$. Optical density at $630 \mathrm{~nm}(\mathrm{~A})$ and neutral lipid production (B) were followed daily for eighteen to twenty days. Error bars represent standard deviations of the triplicates.
\end{abstract}

\section{Figure 2: Final Biomass Yields of strains PCH02 and PCH05 as a Function of Initial Nitrate Concentration}

Both strains were cultured in BBM under mixotrophic and autotrophic conditions with different initial nitrate concentrations as shown. The final biomass yields, in $\mathrm{g} \mathrm{L}^{-1}$ are shown for strain PCH02 (A) and strain PCH05 (B). Biological triplicates were used throughout. Error bars represent standard deviations of the triplicates.

Figure 3. Biomass production of strain $\mathrm{PCH05}$ as a function of nitrate concentration 
Final biomass of strain PCH05 under both mixotrophic and autotrophic conditions is shown as a function of initial nitrate concentrations. Shown is a straight line fit with the slope and correlation coefficients indicated on the graph.

Figure 4: Lipid productivities of strains PCH02 and PCH05 as a Function of Initial Nitrate Concentration

Both strains were cultured in BBM under mixotrophic and autotrophic conditions with different initial nitrate concentrations as shown. The lipid productivities, in $\mathrm{mg} \mathrm{L}^{-1} \mathrm{~d}^{-1}$, calculated for the total length of the incubation, are shown for strain PCH02 (A) and strain PCH05 (B). Biological triplicates were used throughout. Error bars represent standard deviations of the triplicates.

\section{Figure 5: Neutral Lipid Content of strains PCH02 and PCH05 at Different Initial Nitrate Concentrations}

Both strains were cultured in BBM under mixotrophic and autotrophic conditions with different initial nitrate concentrations as shown. The final neutral lipid content, in $\%$ dry weight, are shown for strain PCH02 (A) and strain PCH05 (B). Biological triplicates were used throughout. Error bars represent standard deviations of the triplicates.

\section{Figure 6: Total Volumetric Neutral Lipid Yields of strains PCH02 and PCH05 as a Function of Initial Nitrate Concentration}

Both strains were cultured in BBM under mixotrophic and autotrophic conditions with different initial nitrate concentrations as shown. The final volumetric lipid yields, in $\mathrm{g} \mathrm{L}^{-1}$, are shown for strain PCH02 (A) and strain PCH05 (B). Biological triplicates were used throughout. Error bars represent standard deviations of the triplicates. 
A
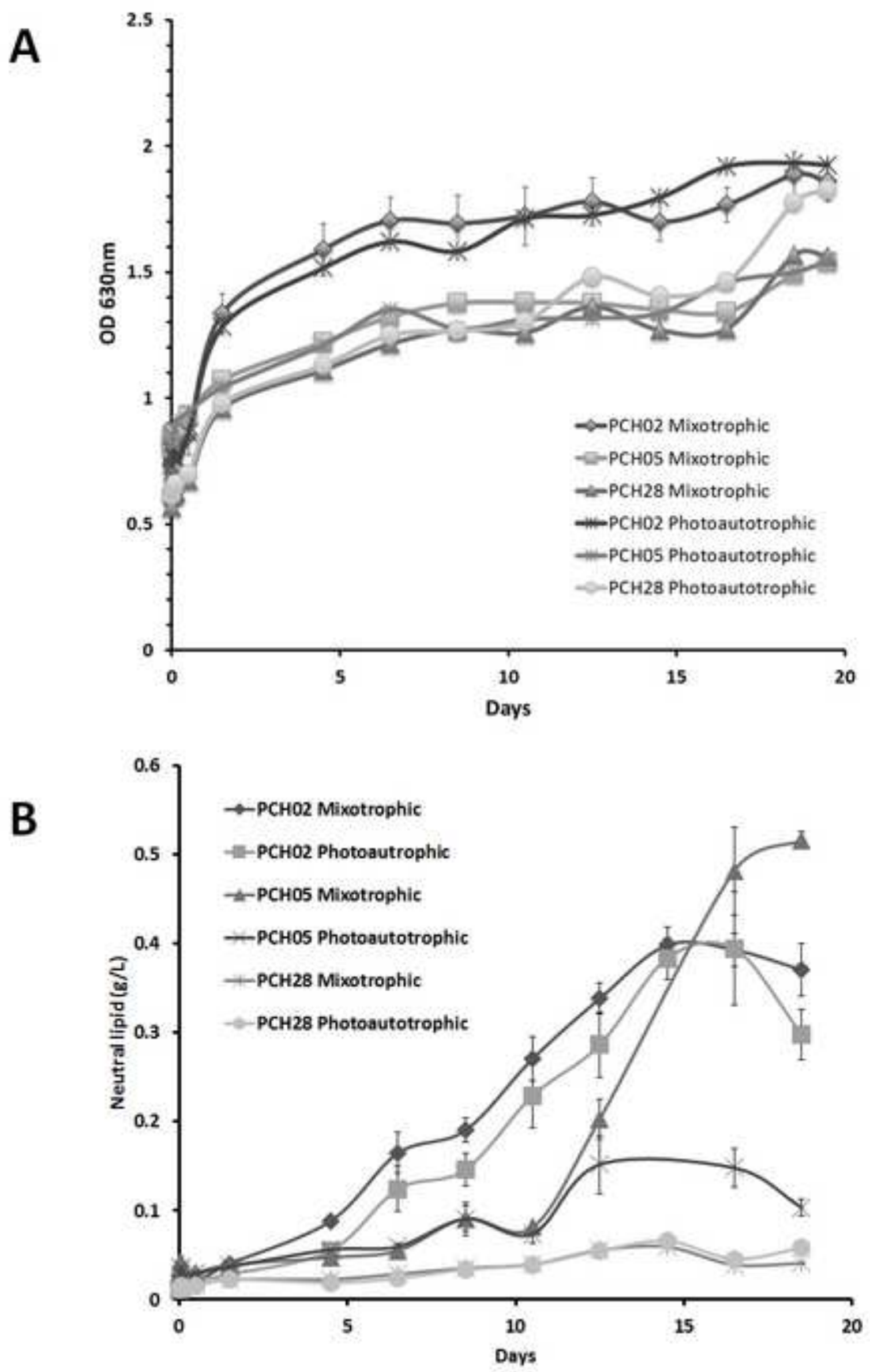


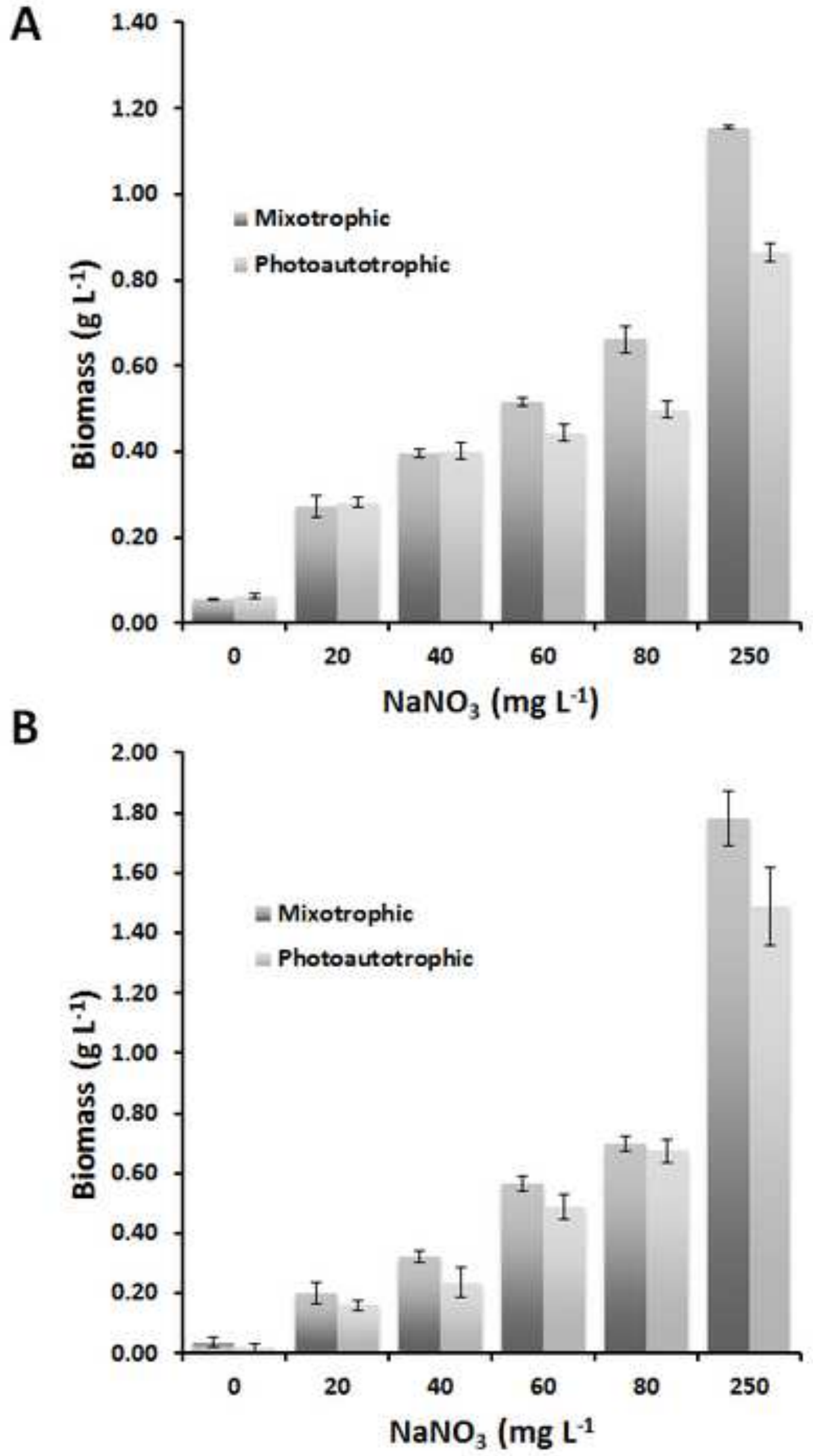




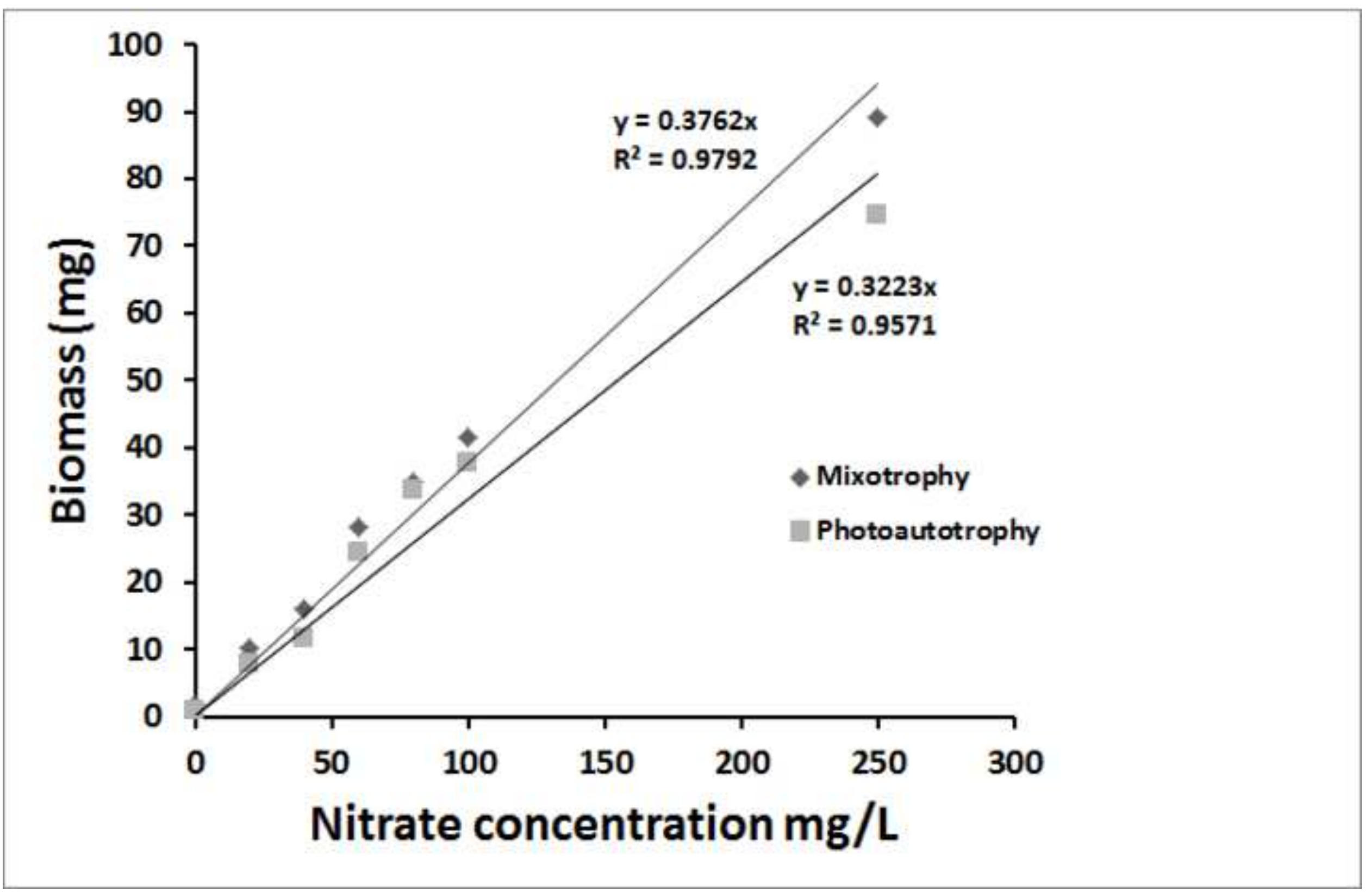



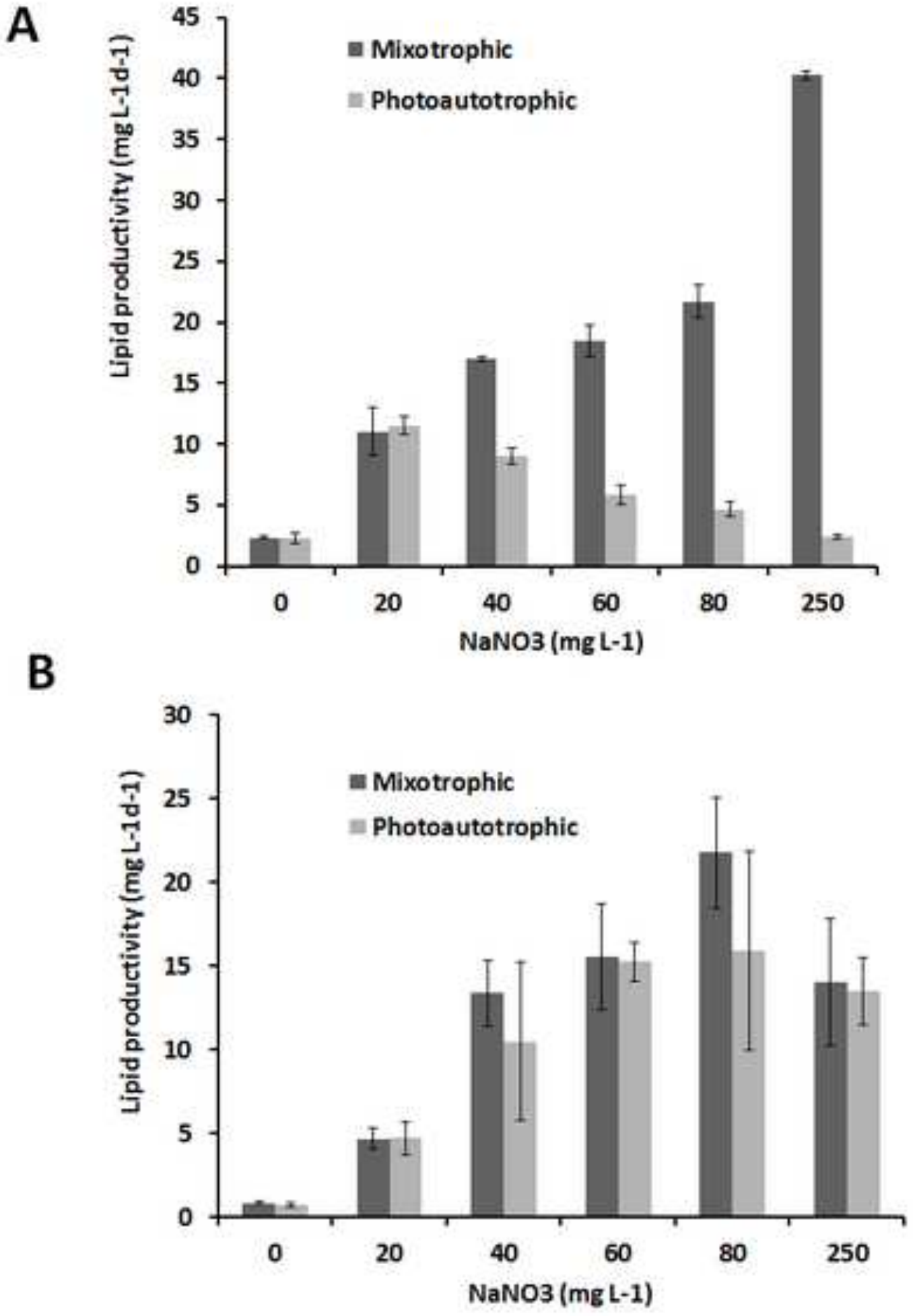

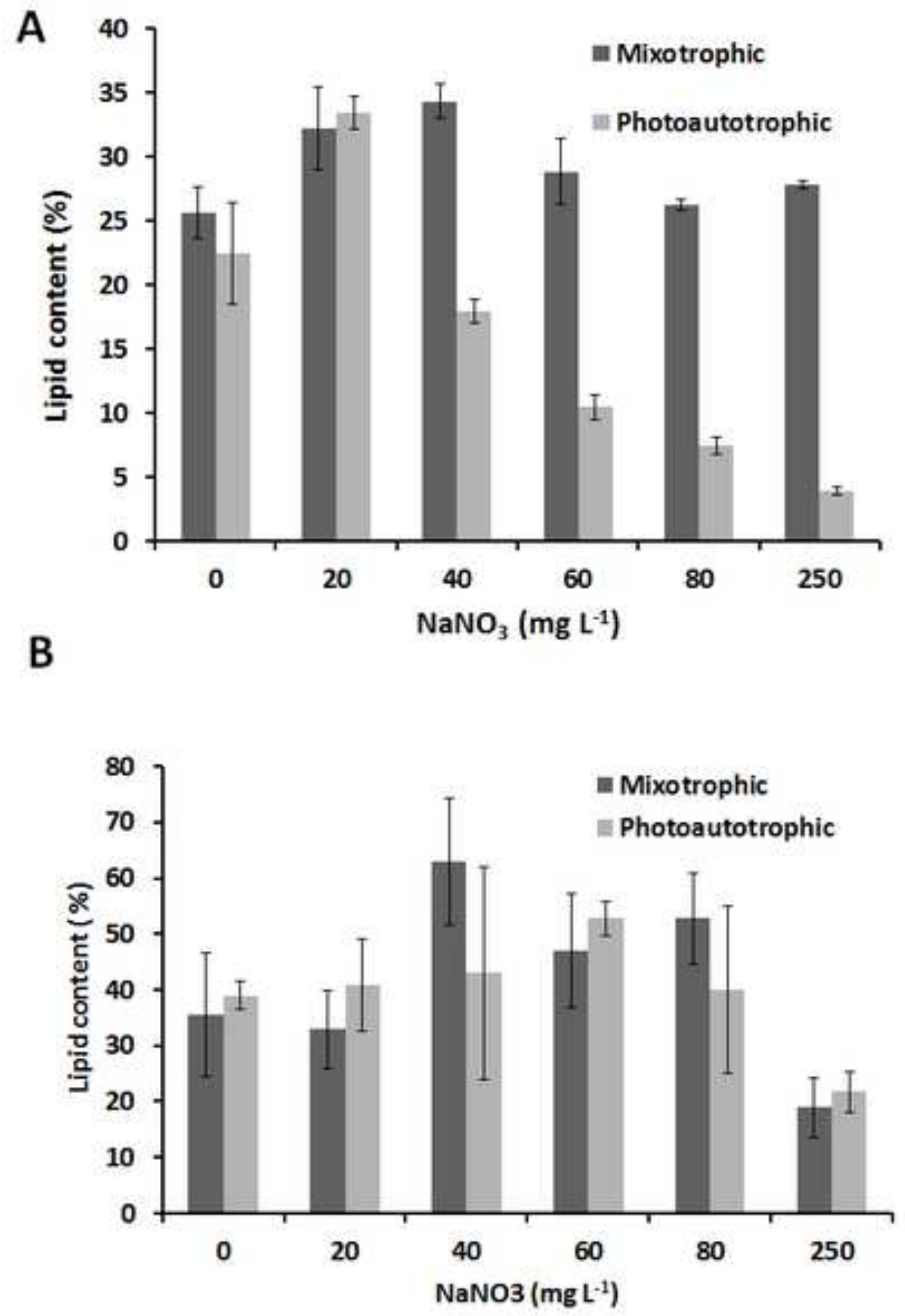

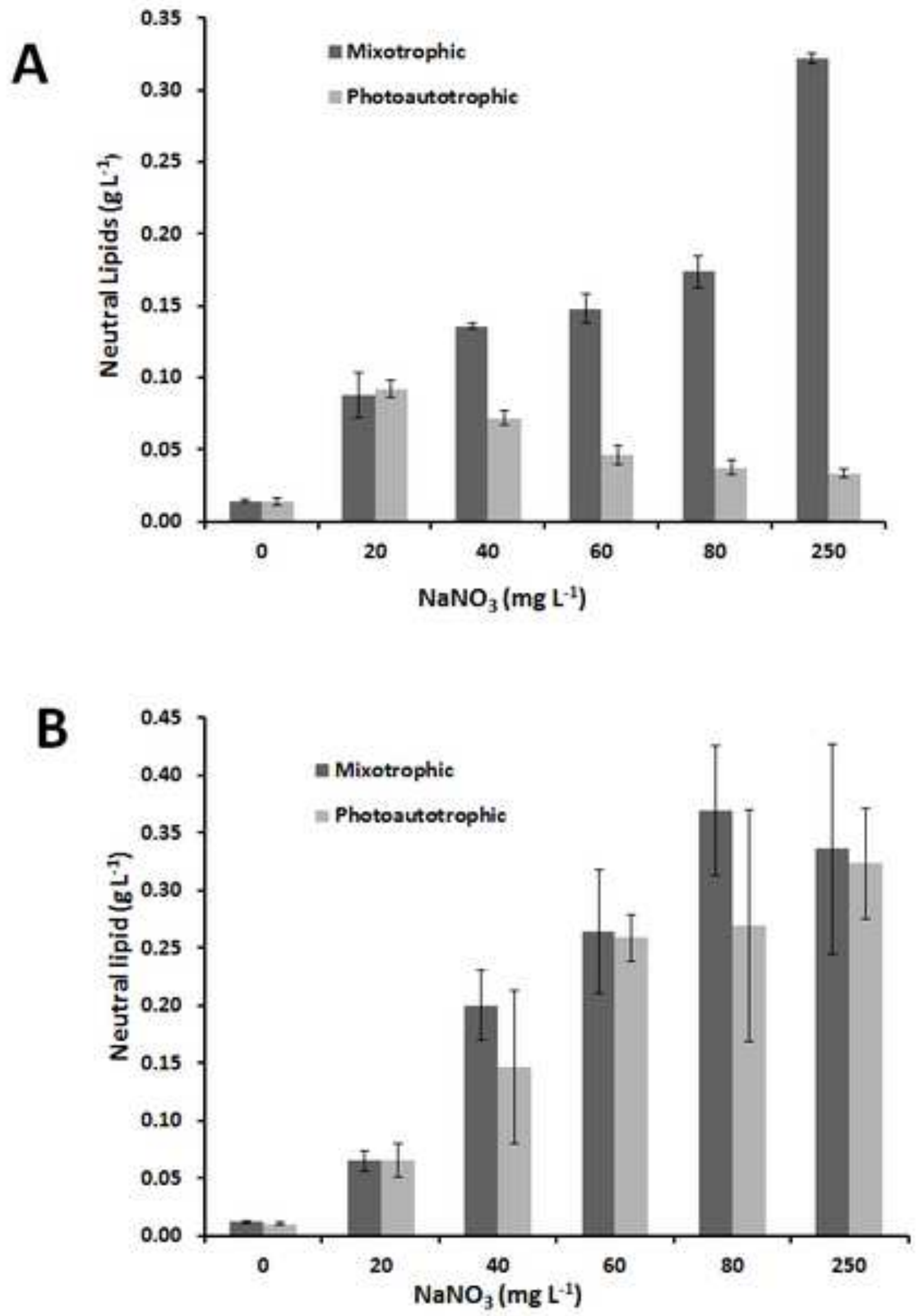
Table 1. Biomass production and growth rates of strains PCH02 and PCH05 grown at different initial nitrate concentrations under mixotrophic and photoautotrophic conditions

\begin{tabular}{|c|c|c|c|c|c|c|c|c|c|c|c|c|}
\hline \multirow[b]{2}{*}{$\begin{array}{l}\mathrm{NaNO}_{3} \\
\left(\mathrm{mg} \mathrm{L}^{-1}\right)\end{array}$} & \multicolumn{3}{|c|}{$\begin{array}{l}\text { Strain PCH02 } \\
\text { Biomass }\left(\mathrm{g} \mathrm{L}^{-1}\right)^{\mathrm{a}}\end{array}$} & \multicolumn{3}{|c|}{ Growth rate $\left(d^{-1}\right)^{b}$} & $\begin{array}{l}\text { Strai } \\
\text { Bioma }\end{array}$ & \multicolumn{4}{|c|}{ Strain PCH05 } & $\left.d^{-1}\right)^{b}$ \\
\hline & $\mathbf{M}^{\mathbf{d}}$ & $\mathbf{P}^{\mathbf{e}^{\mathrm{o}}}$ & Fold & $\mathbf{M}$ & $\mathbf{P}$ & Fold & $\mathbf{M}$ & $\mathbf{P}$ & Fold & $\mathbf{M}$ & $\mathbf{P}$ & Fold \\
\hline 0 & 0.055 & 0.062 & 0.89 & 0.440 & 0.525 & 0.84 & 0.036 & 0.020 & 1.8 & 0.337 & 0.325 & 1.04 \\
\hline 20 & 0.272 & 0.281 & 0.97 & 0.817 & 0.782 & 1.04 & 0.202 & 0.160 & 1.26 & 0.601 & 0.612 & 0.98 \\
\hline 40 & 0.397 & 0.401 & 0.99 & 0.895 & 0.733 & 1.22 & 0.320 & 0.236 & 1.36 & 0.611 & 0.646 & 0.95 \\
\hline 60 & 0.515 & 0.444 & 1.16 & 1.032 & 0.756 & 1.37 & 0.564 & 0.489 & 1.15 & 0.597 & 0.649 & 0.92 \\
\hline 80 & 0.662 & 0.498 & 1.33 & 1.062 & 0.760 & 1.40 & 0.700 & 0.676 & 1.03 & 0.667 & 0.663 & 1.01 \\
\hline 250 & 1.556 & 0.864 & 1.34 & 1.371 & 0.832 & 1.65 & 1.784 & 1.493 & 1.19 & 0.653 & 0.664 & 0.98 \\
\hline
\end{tabular}

\footnotetext{
${ }^{a}$ Determined with triplicates. Standard deviations were omitted to keep the table simpler. Average standard deviation was $4.8 \%$ (M) and $7.2 \%(\mathrm{P})$

${ }^{b}$ Determined with triplicates. Standard deviations were omitted to keep the table simpler. Average standard deviation was $6.5 \%$

${ }^{\mathrm{c}}$ Determined with triplicates. Standard deviations were omitted to keep the table simpler. Average standard deviation was 3.9\% (M) and $4.7 \%(\mathrm{P})$

${ }^{\mathrm{d}} \mathrm{M}$, mixotrophic

${ }^{\mathrm{e}} \mathrm{P}$, photoautotrophic
} 
Table 2: Glycerol consumption and volumetric biomass production for growth under mixotrophic or photoautotrophic conditions

\begin{tabular}{lllll} 
Strains & \multicolumn{2}{c}{ Biomass $\left(\mathrm{g} \mathrm{L}^{-\mathbf{1}}\right)$} & Glycerol consumption \\
& $\mathbf{M}^{\mathbf{a}}$ & $\mathbf{P}^{\mathrm{b}}$ & Fold & $(\boldsymbol{\%})$ \\
PCH02 & 2.46 & 2.36 & 1.04 & 51.25 \\
$\mathbf{P C H 0 5}$ & 2.55 & 2.40 & 1.06 & 47.43 \\
$\mathbf{P C H 2 8}$ & 1.35 & 2.12 & 0.64 & 55.02 \\
${ }^{\mathrm{a}} \mathrm{M}$, mixotrophic & & & \\
${ }^{\mathrm{b}} \mathrm{P}$, photoautotrophic & & &
\end{tabular}

